SNUTP 94-134

hep-ph/9505420

\title{
Light-Front View of The Axial Anomaly
}

\author{
Chueng-Ryong $\mathrm{Ji}^{1,3}$ and Soo-Jong Rey ${ }^{2}$ \\ Department of Physics, North Carolina State University \\ Raleigh, North Carolina 27695-8202 USA ${ }^{1}$ \\ Department of Physics $\&$ Center for Theoretical Physics \\ Seoul National University, Seoul 151-742, Korea ${ }^{2}$ \\ Institute for Nuclear Theory, University of Washington \\ Seattle, Washington $98195^{3}$
}

(March 4, 2018)

\begin{abstract}
Motivated by an apparent puzzle of the light-front vacua incompatible with the axial anomaly, we have considered the two-dimensional massless Schwinger model for an arbitrary interpolating angle of the quantization surface. By examining spectral deformation of the Dirac sea under an external electric field semiclassically, we have found that the axial anomaly is quantization angle independent. This indicates an intricate nontrivial vacuum structure present even in the light-front limit.
\end{abstract}

11.15.-q,11.30.Rd,12.20.-m

Typeset using REVTEX 
Recently the idea of using light-front quantization [1], which has been applied successfully in the context of current algebra [2] and parton model [3] in the past, was revived as a promising method for solving QCD [4,5]. While the hope is partly based on the observation that the perturbative vacuum becomes extremely simple, there has never been any serious study of nontrivial vacuum states such as chiral symmetry breaking and $\theta$-vacuum structures. As these aspects are essential to the low-energy hadron physics, it is important to understand how these aspects come about in the light-front quantized QCD. In simpler models, this issue has been studied only very recently [6], and found that the $k^{+}=0$ zero-modes are responsible for nontrivial vacuum phenomena.

In this Letter, we address another particular aspect of the nontrivial vacuum structure: the axial anomaly [7]. It is well-known that regularization procedure in quantum field theory sometimes cannot preserve all of the classical symmetries. The axial anomaly represents a paramount example of such phenomena. The axial anomaly may be understood as an ultraviolet phenomena stemming from the linear divergence of the one-loop diagrams. Since the diagram is divergent, one has to regularize, for example, through Pauli-Villar method. It is also known that the axial anomaly can be understood as an infrared phenomenon arising from the level crossing of the Dirac vacuum in the background of nontrivial gauge fields. The level crossing is nothing but a pair creation of the charged fermions. This interpretation is apparently incompatible with the light-front quantization since the fermion pair creation is not possible due to the $k^{+}$momentum conservations. It is therefore the purpose of this letter to see how the light-front quantized fermionic vacuum responds to produce the axial anomaly phenomena. For simplicity, we will only consider the two-dimensional Schwinger model [8].

We study the massless Schwinger model quantized with an interpolating coordinates [9] $\left(x^{+}, x^{-}\right)$,

$$
\left(\begin{array}{l}
x^{+} \\
x^{-}
\end{array}\right) \equiv\left(\begin{array}{cc}
\sin \theta / 2 & \cos \theta / 2 \\
\cos \theta / 2 & -\sin \theta / 2
\end{array}\right)\left(\begin{array}{l}
t \\
x
\end{array}\right) .
$$

The $x^{+}, x^{-}$are taken as time and space coordinates respectively. These coordinates inter- 
polate between the equal-time one at $\theta=\pi$ and the light-front one at $\theta=\pi / 2$. For an arbitrary interpolating angle $\theta$ we denote $c \equiv-\cos \theta$ and $s \equiv \sin \theta$. In what follows, we put the space coordinate $x^{-}$on a compact circle of circumference $L$ to regulate the infrared limit. The action of the Schwinger model is given by

$$
\mathcal{S}=\int d x^{+} d x^{-}\left[-\frac{1}{4} F_{\mu \nu} F^{\mu \nu}+\bar{\psi} i \gamma^{\mu}\left(\partial_{\mu}+i e A_{\mu}\right) \psi\right]
$$

Here, $\psi$ denotes the two-component Dirac spinor and $\bar{\psi} \equiv \psi^{\dagger} \gamma^{0}$. We choose the Dirac matrices exclusively in the chiral representation. At an arbitrary interpolating angle the Dirac matrices in the chiral representation are given by

$$
\gamma^{ \pm}=\left(\begin{array}{cc}
0 & \pm \sqrt{1 \pm s} \\
\sqrt{1 \mp s} & 0
\end{array}\right) .
$$

They satisfy Dirac matrix algebra $\left\{\gamma^{ \pm}, \gamma^{ \pm}\right\}=2 g^{ \pm \pm} \mathbf{I}= \pm 2 c \mathbf{I}$ and $\left\{\gamma^{+}, \gamma^{-}\right\}=2 g^{+-} \mathbf{I}=2 s \mathbf{I}$. At equal-time limit $c \rightarrow 1$ and $s \rightarrow 0, \gamma^{ \pm}$become $\gamma^{0}=\sigma^{1}$ and $\gamma^{1}=i \sigma^{2}$ respectively. Note that the (anti)-Hermiticity property $\gamma^{0 \dagger}=+\gamma^{0}, \gamma^{1 \dagger}=-\gamma^{1}$ is valid only at equal-time limit. For a generic interpolating angle $\gamma^{ \pm}$are neither Hermitian nor anti-Hermitian; they are related each other through $\gamma_{5} \equiv \gamma_{o} \gamma_{1}=\sigma_{3}$ as $\gamma^{ \pm \dagger}=\mp \gamma^{\mp} \gamma_{5}$. More generally $\gamma_{5}$ defined as $\gamma_{5} \equiv\left(\gamma^{+} \gamma^{-}-\gamma^{-} \gamma^{+}\right) / 2=\sigma_{3}$, independent of the interpolating angle $\theta$. Therefore it is always possible to decompose the Dirac fermion into LH and RH components for an arbitrary angle $\theta$

$$
\psi=\left(\begin{array}{c}
\psi_{L} \\
\psi_{R}
\end{array}\right)=\frac{1}{\sqrt{L}} \sum_{n}\left(\begin{array}{c}
\psi_{L n} \mathbf{a}_{L n} \\
\psi_{R n} \mathbf{a}_{R n}
\end{array}\right) e^{i p_{n} x^{-}} ; \quad p_{n}=2 \pi n / L
$$

The fermions satisfy an anti-periodic boundary condition $\psi\left(x^{-}+L\right)=-\psi\left(x^{-}\right)$. Hence, the momentum quantum number is half-integer valued $n \in \mathbf{Z}+1 / 2$. Free fermions of each chirality satisfies the following equations of motion

$$
\begin{aligned}
& {\left[c \partial_{+}+(1+s) \partial_{-}\right] \psi_{L}\left(x^{+}, x^{-}\right)=0} \\
& {\left[(1+s) \partial_{+}-c \partial_{-}\right] \psi_{R}\left(x^{+}, x^{-}\right)=0 .}
\end{aligned}
$$

Solving the above equations of motion, the basis wave functions $\psi_{L n}, \psi_{R n}$ in Eq.(4) are given by 


$$
\begin{aligned}
& \psi_{L n}=\theta(-n)(1-s)^{-1 / 4}, \\
& \psi_{R n}=\theta(+n)(1+s)^{-1 / 4} .
\end{aligned}
$$

The canonical quantization then proceeds with an anticommutation relation $\left\{\pi_{\psi}(x), \psi(y)\right\}_{x^{+}=y^{+}}=i \delta\left(x^{-}-y^{-}\right)$where $\pi_{\psi}(x)=\partial \mathcal{L} / \partial\left(\partial_{+} \psi(x)\right)=i \bar{\psi}(x) \gamma^{+}$. In terms of chiral fermion components, $\sqrt{1-s}\left\{\psi_{L}^{\dagger}(x), \psi_{L}(y)\right\}_{x^{+}=y^{+}}=\sqrt{1+s}\left\{\psi_{R}^{\dagger}, \psi_{R}(y)\right\}_{x^{+}=y^{+}}=$ $\delta\left(x^{-}-y^{-}\right)$. Consequently the mode creation and annihilation operators satisfy $\left\{\mathbf{a}_{L n}, \mathbf{a}_{L m}^{\dagger}\right\}=\left\{\mathbf{a}_{R n}, \mathbf{a}_{R m}^{\dagger}\right\}=\delta_{n, m}$ and $\left\{\mathbf{a}_{L n}, \mathbf{a}_{R m}\right\}=0$. For the gauge field we choose a gauge $\partial_{-} A_{-}=0$ gauge. Not only permitting a manifestly periodic boundary condition for $A_{+}$, this gauge choice has an advantage of representing a constant electric field as $\partial_{+} A_{-}^{(o)}\left(x^{+}\right)$ where $A_{-}^{(o)}\left(x^{+}\right)$is the $x^{-}$independent, zero mode of $A_{-}$. The canonical momentum conjugate to $A_{-}^{(o)}, \Pi_{o}=\int d x^{-} \partial \mathcal{L} / \partial\left(\partial_{+} A_{-}^{(o)}\right)=L \partial_{+} A_{-}^{(o)}$ satisfies the usual commutation relation $\left[\Pi_{0}\left(x^{+}\right), A_{-}^{(o)}\left(x^{+}\right)\right]=-i$.

Hamiltonian of the Schwinger model projected to gauge zero-mode is expressed as

$$
\begin{aligned}
P_{+}^{(o)} & =\Pi_{0} \partial_{+} A_{-}^{(o)}+\int d x^{-}\left(\pi_{\psi} \partial_{+} \psi-\mathcal{L}\right) \\
& =\frac{L}{2} E^{2}+\int d x^{-}\left[-\sqrt{1+s} \psi_{L}^{\dagger} i \partial_{-} \psi_{L}+\sqrt{1-s} \psi_{R}^{\dagger} i \partial_{-} \psi_{R}+e A_{-}^{(o)} J^{-}+e A_{+} J^{+}\right] .
\end{aligned}
$$

The gauge currents $J^{ \pm}$are related to the chiral currents $J_{L, R} \equiv \sqrt{1 \mp s} \psi_{L, R}^{\dagger} \psi_{L, R}$ as $J^{ \pm}=$ $\sqrt{1 \mp s} \psi_{L}^{\dagger} \psi_{L} \pm \sqrt{1 \pm s} \psi_{R}^{\dagger} \psi_{R}$ so that $J^{+}=J_{L}+J_{R}, J^{-}=(1+s) / c J_{L}-(1-s) / c J_{R}$. As it stands, however, $J^{ \pm}$are ill-defined because of short-distance singularity of composite operators. One may define them in terms of the currents regularized by Schwinger's pointsplitting method along the $x^{-}$space direction

$$
J^{ \pm}[\epsilon] \equiv \bar{\psi}\left(x^{-}+\epsilon\right) \gamma^{ \pm} e^{-i e \int_{x^{-}}^{x^{-}+\epsilon} d x^{-} A_{-}^{(0)}\left(x^{+}\right)} \psi\left(x^{-}\right)=\bar{\psi}\left(x^{-}+\epsilon\right) \gamma^{ \pm} e^{-i e \epsilon A_{-}^{(o)}\left(x^{+}\right)} \psi\left(x^{-}\right)
$$

Using the short-distance behavior of the fermion bilinear $\psi_{L, R}^{\dagger} \psi_{L, R} \rightarrow \pm i / 2 \pi \epsilon \sqrt{1 \mp s}$, it is straightforward to evaluate the regularized currents as

$$
J^{+}=: J^{+}:, \quad J^{-}=: J^{-}:+\frac{e}{\pi c} A_{-}^{(o)}
$$


Thus, gauge invariant regularization leads to an important modification of the Hamiltonian $P_{+}^{(o)}$ for the quantum mechanics of $A_{-}^{(o)}$ :

$$
P_{+}^{(o)}=\frac{1}{2 L} \Pi_{0}^{2}+e A_{-}^{(o)}\left(\frac{s}{c}: Q:+\frac{1}{c}: Q_{5}:\right)+\frac{e^{2}}{\pi c} L\left(A_{-}^{(o)}\right)^{2}
$$

in which $Q_{L, R} \equiv \int d x^{-} J_{L, R}$ and $Q=Q_{L}+Q_{R}, Q_{5}=Q_{L}-Q_{R}$. The Heisenberg equation of motion for the zero-mode $A_{-}^{(o)}$ then reads

$$
L \partial_{+}^{2} A_{-}^{(o)}=-e\left(\frac{s}{c}: Q:+\frac{1}{c}: Q_{5}:\right)-\frac{2 e^{2}}{\pi c} L A_{-}^{(o)} .
$$

Because of the gauge invariance $A_{+} \rightarrow A_{+}+\partial_{+} \Lambda$ the electric charge is manifestly conserved $d Q / d x^{+}=0$. On the other hand, for a constant electric field background $E=-\partial_{+} A_{-}^{(o)}, x^{+}$-derivative of the equation of motion for $A_{-}^{(o)}$ becomes

$$
\partial_{+} Q_{5}=\frac{2 e}{\pi} L E
$$

viz. the axial charge is anomalously produced at a rate proportional to the constant electric field $E$. This is precisely the axial anomaly in the massless Schwinger model.

In the above method, the axial anomaly has arisen from regularizing short-distance singularities of coincident quantum operators. As such, the axial anomaly may be interpreted as a ultraviolet phenomena. Alternative method is a direct calculation of the relevant Feynman diagrams. At the light-front limit, the axial anomaly was calculated in this way [10]. However the calculation was rather involved compared to the perturbative calculation in the equal-time limit. On the other hand it is well-known that the axial anomaly may be understood in yet another, semiclassical way [11] through spectral flow [12] crossing the zero-energy level in the Dirac vacuum and simultaneous pair production of left- and rightmoving fermions under appropriate external gauge field. This alternative interpretation emphasizes that the nontrivial structure of the Dirac vacuum and that the axial anomaly is rather an infrared phenomenon. However, this interpretation of the axial anomaly poses a serious interpretational problem at a first glance since Eq.(5) indicates that half of the fermion degrees of freedom decouple at the light-front limit $c=0$. It is not clear at all how the anomaly is understood as a pair production of fermion pairs of opposite chirality. 
In order to resolve this difficulty, we first construct the Dirac vacuum at an arbitrary interpolating angle $\theta$. We define the Dirac vacuum by filling all the negative energy levels of the left- and right-moving fermions to the Fock vacuum:

$$
\mid \text { vac. }\rangle \equiv \prod_{\{n \leq-1 / 2\}} \mathbf{a}_{L,+n}^{\dagger} \mathbf{a}_{R,-n}^{\dagger}|0\rangle .
$$

As is clear the Dirac vacuum is not evenly populated between the left and the right moving chiral modes of fermion. This leads to an important consequence for correctly accounting for the axial anomaly and the vacuum energy.

Chiral charge operators are defined by Eqs. $(12,13)$. In terms of mode operators they are given by

$$
\begin{aligned}
Q_{L} & \equiv \int d x^{-} J_{L}:=: \sum_{n, L H} \mathbf{a}_{L n}^{\dagger} \mathbf{a}_{L n}, \\
Q_{R} & \equiv \int d x^{-} J_{R}:=: \sum_{n, R H} \mathbf{a}_{R n}^{\dagger} \mathbf{a}_{R n} .
\end{aligned}
$$

Let us calculate vacuum chiral charges in an external electric field by evaluating expectation values of $Q_{L, R}$ over the Dirac vacuum. As they stand, the vacuum chiral charges are formally infinite, hence, ill-defined. We therefore define regularized chiral charges by cutting off the ultraviolet negative modes. In order to preserve the gauge invariance, the regularization procedure has to preserve the gauge invariance. Thus

$$
\begin{aligned}
\left\langle Q_{L}(\text { reg. })\right\rangle & =\sum_{n \leq-1 / 2} 1 \cdot \exp \left[\epsilon \frac{1+s}{c}\left(+p_{n}-e A_{-}^{(o)}\left(x^{+}\right)\right)\right], \\
\left\langle Q_{R}(\text { reg. })\right\rangle & =\sum_{n \geq+1 / 2} 1 \cdot \exp \left[\epsilon \frac{c}{1+s}\left(-p_{n}+e A_{-}^{(o)}\left(x^{+}\right)\right)\right] .
\end{aligned}
$$

Here, $\epsilon$ is the regularization parameter and $p_{n}=2 \pi n / L$ for half-integer-valued $n$. Physical quantities will be defined by a finite contribution of the regularized quantities as the regulator is removed $\epsilon \rightarrow 0$. Note that we have introduced the exponential regularization factor which depends explicitly on the interpolating angle $\theta$. This means we need to regularize in an asymmetric manner between the left- and the right-moving fermions. We will see in the following why this is the correct regularization scheme. For now we note that, at equal- 
time limit $\theta \rightarrow \pi$, the proposed regularization Eq.(15) coincides with the one considered by Manton and Shifman [13].

On the other hand, in order to take the light-front limit $\theta \rightarrow \pi / 2$, one has to be careful for the limiting procedure of taking $\epsilon$ and $c \rightarrow 0$. As is clear from the regularized chiral charges $Q_{L, R}$, one has to take $\epsilon$ first to zero before $c \rightarrow 0$ is taken. Only in this limit both the left- and the right-chiral charges are regularized suitably.

It is straightforward to evaluate the regularized charges and expand them in powers of $\epsilon$

$$
\begin{aligned}
& \left\langle Q_{L}(\mathrm{reg})\right\rangle=\frac{L}{\pi} \frac{1}{\epsilon}\left(\frac{c}{1+s}\right)-\frac{e}{\pi} L A_{-}^{(o)}+\frac{\epsilon}{2}\left(\frac{1+s}{c}\right)\left[\frac{L}{\pi}\left(e A_{-}^{(o)}\right)^{2}-\frac{1}{24} \frac{\pi}{L}\right]+\mathcal{O}(\epsilon)^{2}, \\
& \left\langle Q_{R}(\mathrm{reg})\right\rangle=\frac{L}{\pi} \frac{1}{\epsilon}\left(\frac{1+s}{c}\right)+\frac{e}{\pi} L A_{-}^{(o)}+\frac{\epsilon}{2}\left(\frac{c}{1+s}\right)\left[\frac{L}{\pi}\left(e A_{-}^{(o)}\right)^{2}-\frac{1}{24} \frac{\pi}{L}\right]+\mathcal{O}(\epsilon)^{2} .
\end{aligned}
$$

The first terms in Eq.(16) are precisely sources of the infinite constant contribution to each charges as the regulator is removed. Therefore we define the physical fermion charges by simply dropping them out. For a constant electric field, $E=-\partial_{+} A_{-}^{(o)}$, we then find that production rates of physical fermion charges are $\epsilon \rightarrow 0$

$$
\frac{d}{d x^{+}}\left\langle Q_{L}(\mathrm{reg})\right\rangle=+\frac{e}{\pi} L E, \quad \frac{d}{d x^{+}}\left\langle Q_{R}(\mathrm{reg})\right\rangle=-\frac{e}{\pi} L E .
$$

Hence,

$$
\frac{d}{d x^{+}}\langle Q(\mathrm{reg})\rangle=0, \quad \frac{d}{d x^{+}}\left\langle Q_{5}(\mathrm{reg})\right\rangle=\frac{e L}{\pi} 2 E .
$$

We see that the electric charge $Q \equiv Q_{L}+Q_{R}$ is manifestly conserved, consistent with gauge invariance of the proposed regulator. On the other hand, axial charge $Q_{5} \equiv Q_{L}-Q_{R}$ is seen anomalous: nonzero chiral charge is produced out of the Dirac vacuum at a rate $2 e L E / \pi$.

This agrees with the results Eq.(12) obtained from Schwinger's point-splitting method. More importantly, the current conservation and the axial anomaly in Eq.(18) are independent of the interpolating angle $\theta$. In the light-front limit, $c \rightarrow 0$, the axial anomaly is correctly reproduced and remains the same as in the equal-time limit. The crucial point in the above regularization is that different regularizations are imposed to the left- and the right-moving chiral fermions. See Eq. (15). The regularization depends on the interpolating angle $\theta$. As 
is clear from the Fig. 1, right-moving fermions has to be kept for arbitrarily deep levels inside the Dirac sea, while for left-moving fermions a sharp damping is needed. This is indeed what happens for the regularized chiral charges in Eq.(15) and the way to keep the gauge invariance, hence, charge conservation in a manifest way for any interpolation angle $\theta$ including the light-front limit $\theta=\pi / 2$.

The spectral flow of Dirac vacuum fermions also influences the dynamics of the gauge field by contributing to the total energy $P_{+}^{(o)}$ of the zero mode $A_{-}^{(o)}$. Let us calculate the contribution coming from the Dirac vacuum

$$
P_{+}^{(o)}=\frac{1}{2 L} \Pi_{0}^{2}+\left\langle P_{+, f e r m}\right\rangle
$$

where $P_{+, \text {ferm }}$ denotes the energy of fermions in a background of gauge field

$$
P_{+, \text {ferm }}=\sqrt{1+s} \psi_{L}^{\dagger}\left[i \partial_{-}-e A_{-}^{(o)}\right] \psi_{L}-\sqrt{1-s} \psi_{R}^{\dagger}\left[i \partial_{-}-e A_{-}^{(o)}\right] \psi_{R}
$$

A short calculation shows

$$
\left\langle P_{+, \text {ferm }}\right\rangle=\sum_{n \leq-1 / 2}\left(\frac{1+s}{1-s}\right)^{1 / 2}\left(p_{n}-e A_{-}^{(o)}\right)-\sum_{n \geq+1 / 2}\left(\frac{1-s}{1+s}\right)^{1 / 2}\left(p_{n}-e A_{-}^{(o)}\right) .
$$

Again the sum is not well-defined as it stands because of contributions from infinitely many modes. Regularizing the energy in a gauge invariant manner in a way similar to the chiral charges, we find that

$$
\begin{aligned}
\left\langle P_{+, \text {ferm }}\right\rangle & =\sum_{n \leq-1 / 2}\left(\frac{1+s}{c}\right)\left(p_{n}-e A_{-}^{(o)}\right) \exp \left[\epsilon \frac{1+s}{c}\left(+p_{n}-e A_{-}^{(0)}\right)\right] \\
& -\sum_{n \geq+1 / 2}\left(\frac{1-s}{c}\right)\left(p_{n}-e A_{-}^{(o)}\right) \exp \left[\epsilon \frac{1-s}{c}\left(-p_{n}+e A_{-}^{(0)}\right)\right] \\
& =\left[\frac{d Q}{d \epsilon}\right]_{\epsilon \rightarrow 0} .
\end{aligned}
$$

We thus find that

$$
\left\langle P_{+, \text {ferm }}\right\rangle=\frac{e^{2}}{\pi c} L\left(A_{-}^{(o)}\right)^{2},
$$

hence, that the total energy Eq.(19) is given by 


$$
P_{+}^{(o)}=\frac{1}{2 L} \Pi_{0}^{2}+\frac{e^{2}}{\pi c} L\left(A_{-}^{(o)}\right)^{2}
$$

This is precisely the same energy of the zero mode gauge field $A_{-}^{(o)}$ as was found from the Schwinger's point-splitting method in Eq.(12). The second term, which originated from the nontrivial Dirac vacuum deformation under the gauge field, provides the dynamically generated mass $e / \sqrt{\pi}$ of the photon zero mode field $A_{+}^{(o)}$.

In this Letter we have shown that both the axial anomaly and the dynamical mass generation of the two-dimensional massless Schwinger model are correctly reproduced from a regularized Dirac vacuum for any interpolating angle including the light-front limit $\theta=$ $\pi / 2$. The more standard viewpoint of the anomaly and the dynamical mass generation was as ultraviolet phenomena. Because of infinitely many quantum states involved, the axial anomaly and the dynamical mass generation may alternatively be understood as infrared phenomena of the response of the nontrivial Dirac vacuum under an external gauge field. Naive light-front quantization, however, loses half of the chiral degrees of freedom, hence, do not admit such infrared interpretation. This is the question we have addressed and resolved in this Letter. Our results indicate that nontrivial structure of the Dirac vacuum persists at the light-front limit so that the correct axial anomaly and the dynamical mass generation still arises semiclassically from fermion pair production in an external electric field. It would be interesting to see if the similar interpretation can be made in four-dimensional QCD. Our result would also provide further insight to the parton interpretation of the spin-dependent deep inelastic scattering [14. These issue are currently under investigation.

C.-R.J. thanks for the hospitality of the Physics Department and the Center for Theoretical Physics at Seoul National University during his visit. S.-J.R. thanks S. Brodsky for useful discussions and hospitality of the Theory Group of SLAC during his visit. C.-R.J. was supported in part by U.S. Department of Energy under the contract No. DE-FG0590ER40589. S.-J.R was supported in part by U.S. NSF-KOSEF Bilateral Grant '94, KRF International Coorporation Grant '94, KRF Nondirected Research '94 Grant, Ministry of Education BSRI-94-2418 and KOSEF-SRC Program. 


\section{REFERENCES}

[1] P.A.M. Dirac, Rev. Mod. Phys. 21 , 392 (1949); P.J. Steinhardt, Ann. Phys. 128 , 425 (1980).

[2] S.Fubini, G. Furlan, Physics 1 , 229 (1965); S. Weinberg, Phys. Rev. 150 , 1313 (1966); J. Jersak and J. Stern, Nucl. Phys. B7 , 413 (1968); H. Leutwyler, in Springer Tracks in Modern Physics Vol. 50, ed. G. Höhler, (Berlin, 1969).

[3] J.D. Bjorken, Phys. Rev. 179 , 1547 (1969); S.D. Drell, D. Levy, T.M. Yan, Phys. Rev. 187,2159 (1969), D1, 1035 (1970).

[4] H.C. Pauli and S.J. Brodsky, Phys. Rev. D32 , 1993, 2001 (1985); S.J. Brodsky and H.C. Pauli, in 'Recent Aspects of Quantum Fields', eds. H. Mitter and H. Gausterer, Lecture Notes in Physics, Vol.396 (Springer, Berlin, 1991).

[5] R.J. Perry, A.Harindranath, K.G. Wilson, Phys. Rev. Lett. 65 , 2959 (1990), Phys. Rev. D43 , 492, 4051 (1991); D. Mustaki, S. Pinsky, J. Shigemitsu and K. Wilson, Phys. Rev. D43 , 3411 (1991).

[6] T. Maskawa and K. Yamawaki, Prog. Theo. Phys. 56 (1976) 270; G.McCartor, Z.Phys.C52, 611(1991); F.Lenz, in Proceedings of the NATO Advanced Summer Institute on Nonperturbative Quantum Field Theory, edited by D.Vautherin, F.Lenz and J.W.Negele(Plenum Press,N.Y.,1990); Th.Heinzl, S.Krusche and E.Werner, Phys.Lett.B256, 55(1991); R.W. Brown, J.-W. Jun, S.M. Shvartsman and C.C. Taylor, Phys. Rev. D48 , 5873 (1993); S.Z. Huang, W. Lin, Ann. Phys. (N.Y.) 226 , 248 (1993); M. Maeno, Phys. Lett. 320B , 83 (1994); A. Kalloniatis, H.-C. Pauli and S. Pinsky, Phys. Rev. D50 , 6633 (1994); A. Kalloniatis and H.-C. Pauli, Z. Phys. C63 , 161 (1994); M. Tachibana, KOBE-TH-95-01 preprint, unpublished (1995).

[7] S. Adler, Phys. Rev. 177 , 2426 (1969); J. Bell and R. Jackiw, Nuovo Cim. 60A , 47 (1969); S.L. Adler and W. Bardeen, Phys. Rev. 184 , 1848 (1969); J. Wess and B. 
Zumino, Phys. Lett. 37B, 95 (1971).

[8] J. Schwinger, Phys. Rev. 128 , 2425 (1962).

[9] K. Hornbostel, Phys. Rev. D45 , 3781 (1992).

[10] D.M. Capper, D.R.T. Jones and M.J. Litvak, Z. Phys. C32 (1986) 221; Q. Ho-Kim, L. Marleau and P. Mathieu, Phys. Rev. D35 (1987) 1429.

[11] R.P. Feynman, Caltech lecture note (unpublished); J.P. Preskill, Caltech lecture note (unpublished); H.B. Nielsen and M. Ninomiya, Phys. Lett. 130B (1983) 389; A. Manohar, Phys. Lett. B (1985) ; A.H. Mueller, Phys. Lett. B234 (1990) 517.

[12] C.G. Callan, R. Dashen and D. Gross, Phys. Rev. D17 , 2717 (1978); J. Kiskis, Phys. Rev. D18 , 3690 (1978); N.H. Christ, Phys. Rev. D21 , 1591 (1980); J. Ambjorn, J. Greensite and C. Petersen, Nucl. Phys. B221 , 381 (1983).

[13] N.S. Manton, Ann. Phys. 159 (1985) 220; M.A. Shifman, Phys. Rep. 209 (1991) 341.

[14] R.D. Carlitz, J.C. Collins and A.H. Mueller, Phys. Lett. B214(1988) 229. 


\section{FIGURES}

FIG. 1. (a) energy-momentum dispersion relation for free fermions at arbitrary interpolating angle. Empty circles are vacant states; filled circles are occupied states. (b) energy-momentum dispersion relation at external electric field. Spectral flows produces vacant negative energy states and filled positive energy states. 

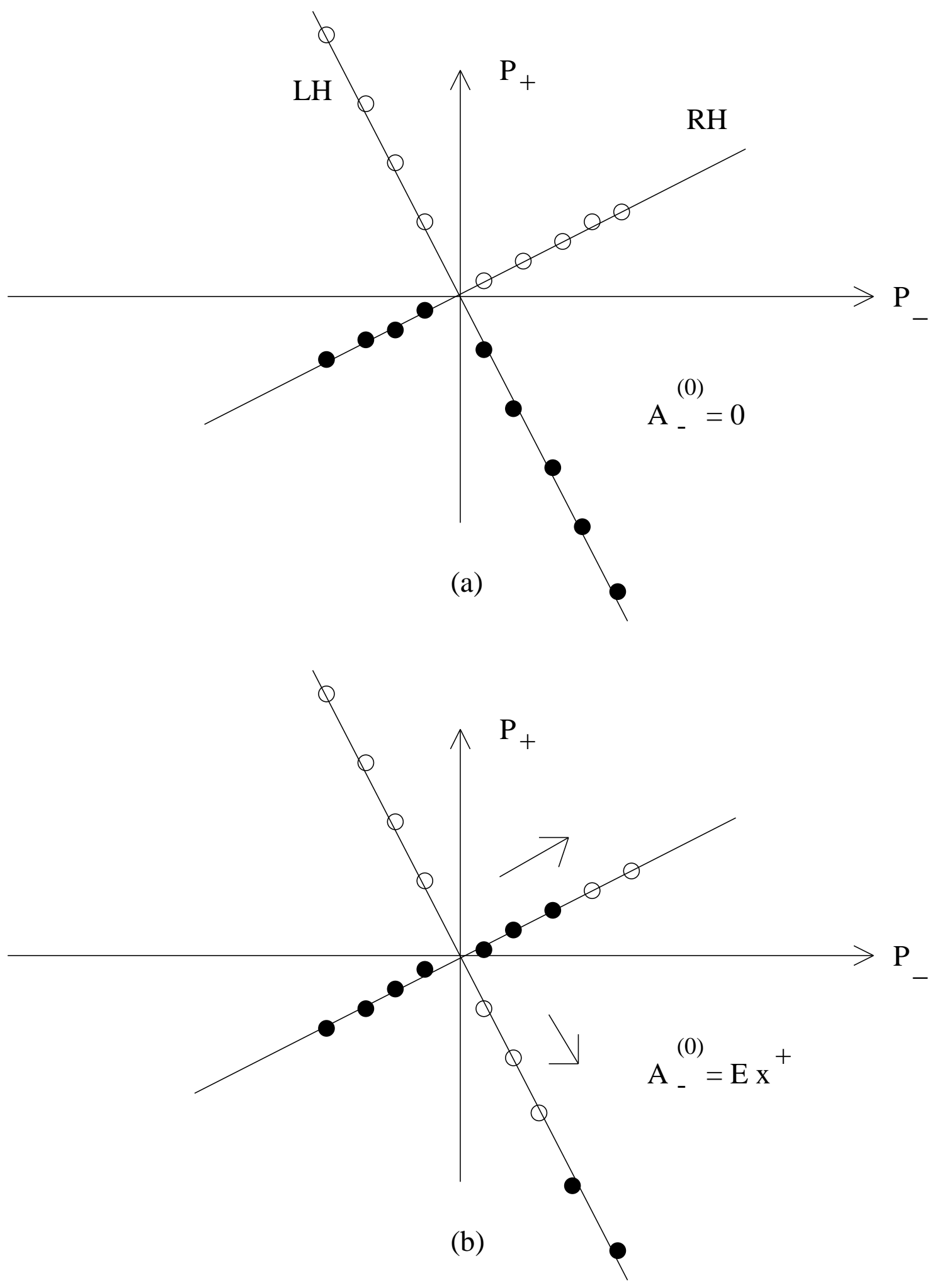